\title{
Trans-radial Coronary Intervention (TCI) using 5-Fr versus 6-Fr guiding catheters in the setting of Acute Coronary Syndrome (ACS)
}

\begin{abstract}
Ahmed Amro, MD', Amal Sobeih, MD², Mohammad Amro, MD', Obadah Aqtash, MD', Hisham Hirzallah, MD', Majd Kanbour, MD', Adee El-Hamdani, MS4' ${ }^{1}$ Alaa Gabi, MD', Yazan Numan, MD', Mark Studeny, MD' ${ }^{1}$, Mehiar El-Hamdani, MD'1
\end{abstract}

\section{ABSTRACT}

BACKGROUND: As in any vascular access the size of guiding catheter is an operator preference. Although multiple studies have showed that the use of 5-Fr and 6-Fr guiding catheters for transradial coronary intervention $(\mathrm{TCl})$ have similar vascular safety profiles, the data comparing the 5-Fr vs 6-Fr guiding catheters for $\mathrm{TCl}$ in terms of fluoroscopy time, procedure time and contrast amount in the setting of acute coronary syndrome (ACS) is limited. We conducted this study to compare the use of 5-Fr versus 6-Fr guiding catheters for $\mathrm{TCl}$ in the settings of ACS.

METHOD: Our study is a single center, retrospective cohort study designed to compare the use of 5-Fr versus 6-Fr guiding catheters for TCl in the setting of ACS. In the period between July 2014 and July 2015, all patients who had previously undergone percutaneous coronary intervention $(\mathrm{PCl})$ with having at least one stent being placed, utilizing a radial access, and using a 5-Fr or 6-Fr guiding catheter were included. No exclusion criteria were applied. The study was approved by Marshall University's institutional review board.

RESULTS: There was a significant reduction in the volume of contrast medium used with the 5-Fr group compared to the 6-Fr group $(130.66+/-3.46 \mathrm{ml}$ vs. $166.25+/-10.05 \mathrm{ml}$ in the 5-Fr and 6-Fr groups, respectively; $\mathrm{p}<0.001)$, fluoroscopy time (12.62 +/- $0.50 \mathrm{~min}$ vs. $16.61+/-1.28 \mathrm{~min}$ in the 5 -Fr and 6-Fr groups, respectively; $p=0.005)$ and there was also significant reduction in the procedure time in the 5-Fr group (38.74 +/- 1.27 min vs. $46.03+/-2.86$ min in the 5-Fr and 6-Fr groups, respectively; $\mathrm{p}=0.023$ ).

CONCLUSION: TCI in the settings of ACS is safe and feasible, whether using 5-Fr or 6-Fr catheters. Our study concluded that using 5-Fr catheters for $\mathrm{TCl}$ could be preferred for patients presenting with ACS due to lower amount of contrast medium used and less fluoroscopy and procedure time. However, this is a single center retrospective study, so we suggest that large randomized controlled studies are needed.

\section{KEYWORDS}

Radial, PCl, Catherization, ACS, Contrast, Fluoroscopy, 5-Fr, 6-Fr, Catheter
Author affiliations are listed at the end of this article.

Correspondence to: Ahmed Amro, MD Marshall University Joan C. Edwards School of Medicine amro@marshall.edu

\section{BACKGROUND}

The radial approach offers multiple advantages for vascular access. The superficial location of the vessel makes access and hemostasis straightforward, with a very low incidence of vascular complications. In most individuals, the presence of dual circulation to the hand via the ulnar artery, which communicates with the radial artery via a palmar arch, minimizes the risk of ischemic complications should radial occlusion occur. The recommended entry point into the radial artery is not close to the nerve and vein, thus limiting the possibility of injury. Because there is no need to lie supine afterward, patient comfort 
and time to ambulation are dramatically improved, and with earlier ambulation times and lower vascular complication rates, hospital costs may be reduced. Dr. Campeau in 1989 reported the first successful diagnostic transradial coronary catheterization. ${ }^{1}$ In later years, it was followed by the first successful transradial coronary intervention, which was conducted by Kiemeneij in 1993. ${ }^{2}$ Subsequently, the radial artery has been proven as the preferred access site for coronary intervention; its many advantages include: lower rates of access site complications, shorter hospital stays, and better patient satisfaction in comparison with transfemoral access. ${ }^{3-5}$ The RIVAL trial, which compared radial versus femoral access for coronary intervention in patients presenting with acute coronary syndromes, showed that radial access in ACS settings was associated significant reduction in major vascular access site complications compared to femoral access. ${ }^{6}$

The disadvantages of radial artery access are the small caliber of the artery that can accommodate only smaller sheath and catheter systems (up to 6-Fr in most patients, and occasionally 7-Fr in a large individual); "slender" sheath systems minimize the outer diameter required to accommodate the corresponding catheters. Radial artery spasm may limit the ability to manipulate catheters and can be very painful.

One of the main complications of radial artery access approach is radial artery occlusion (RAO). The incidence of RAO varies widely in literature with some studies reporting low incidence $(\sim 1 \%)$ and some studies reporting almost one third of patients will have RAO. The high variation in the incidence is most likely because RAO is overlooked as most of the operators do not check for radial artery patency before discharge. ${ }^{7}$

Although multiple studies have shown that the use of $5-\mathrm{Fr}$ versus 6 - $\mathrm{Fr}$ guiding catheters for $\mathrm{TCl}$ have similar vascular safety profiles, ${ }^{8,9}$ the data comparing the 5-Fr vs 6-Fr guiding catheters for $\mathrm{TCl}$ in terms of fluoroscopy time, procedure time and contrast amount in the setting of ACS is limited.

\section{METHODS}

\section{StUdY DESIGN AND PATIENT POPULATION}

Our study is a single center, retrospective cohort study designed to compare the use of 5-Fr versus 6-Fr guiding catheters for $\mathrm{TCl}$ in the setting of ACS. In the period between July 2014 and July 2015, all patients who had previously undergone $\mathrm{PCl}$ with having at least one stent being placed, utilizing a radial access, and using a 5-Fr or 6-Fr guiding catheter were included. No exclusion criteria were applied. The study was approved by Marshall University's institutional review board.

\section{Procedure Description}

Transradial catheterization was performed via the right or left radial artery as per operator preference. A 5-Fr or 6-Fr guiding catheter was then used. Radial mix including heparin, nitroglycerin and verapamil was administered to prevent arterial spasm and thrombosis. At the end of the procedure a transradial band was applied to achieve hemostasis.

\section{ENDPOINTS}

The primary study endpoint was the contrast amount, fluoroscopy time and procedure time which is defined as the interval between administration of local anesthesia for obtaining vascular access and removal of the last catheter.

\section{Data Collection}

Demographics and clinical characteristics of patients along with the various endpoints of the study were retrospectively collected from medical records as detailed in Table 1.

\section{StATISTICAL ANALYSIS}

Categorical variables were presented as percentages and compared using the $T$ score test as appropriate. Contrast volume administered during the procedure, fluoroscopy time and total procedure time are 


\begin{tabular}{|c|c|c|c|}
\hline & Overall $n=267$ & 5 Fr group $n=203$ & $6 \mathrm{Fr}$ group $n=64$ \\
\hline Male n (\%) & $175(65.5 \%)$ & $126(62.0 \%)$ & $49(76.6 \%)$ \\
\hline Female n (\%) & $92(34.5 \%)$ & $77(38 \%)$ & $15(23.4 \%)$ \\
\hline Age mean (SD) & $65.1(11.8)$ & $65.4(12.1)$ & $64.5(11.1)$ \\
\hline Height $\mathrm{cm}$ mean (SD) & $172.9(10.9)$ & $172.6(11.0)$ & $173.7(10.8)$ \\
\hline Weight Kg mean (SD) & $93.5(25.0)$ & $92.5(23.5)$ & $96.2(29.6)$ \\
\hline $\begin{array}{l}\text { Body Mass Index mean } \\
\text { (SD) }\end{array}$ & $31.2(7.9)$ & $31.1(7.6)$ & $31.7(9.1)$ \\
\hline History of CABG & $23(8.6 \%)$ & $17(8.3 \%)$ & $6(9.3 \%)$ \\
\hline Number of stents (SD) & $1.3(0.8)$ & $1.3(0.8)$ & $1.3(0.9)$ \\
\hline $\begin{array}{l}\text { Presentation as STEMI n } \\
(\%)\end{array}$ & $14(5.2 \%)$ & $9(4.4 \%)$ & $5(7.8 \%)$ \\
\hline $\begin{array}{l}\text { Presentation as NSTEMI } \\
\mathrm{n}(\%)\end{array}$ & $112(41.9 \%)$ & $82(40.4 \%)$ & $30(46.9 \%)$ \\
\hline $\begin{array}{l}\text { Presentation as UA n } \\
(\%)\end{array}$ & $141(52.8 \%)$ & $113(55.6 \%)$ & $28(43.7 \%)$ \\
\hline
\end{tabular}

TABLE 1: Patients demographics and characteristics

continuous variables and they were presented as mean \pm SD. All analyses were done using SPSS version 24.0. statistical software. Differences were assumed statistically significant when $P$ value $<0.05$.

\section{RESULTS}

From July 2014 through July 2015, a total of 267 transradial catheterization procedures were performed at our center. The 5-Fr guiding catheter was used in 203 patients while the 6-Fr guiding catheter was used in 64 patients. The mean patient age of the overall sample was $65.1 \pm 11.8$ years; $34.5 \%$ of which were females and $65.5 \%$ were males. Presentation as ST-segment elevation myocardial infraction (STEMI) was seen in 5.2\%, non-STsegment elevation myocardial infarction (NSTEMI) in $41.9 \%$, and unstable angina (UA) in $52.8 \%$. The two groups' characteristics are expressed in Table I. There was a significant reduction in the volume of contrast medium used with the 5-Fr group compared to the 6-Fr group (130.66 +/- $3.46 \mathrm{ml}$ vs. $166.25+/-10.05 \mathrm{ml}$ in the 5-Fr and 6-Fr groups, respectively; $\mathrm{p}<0.001$ ), fluoroscopy time (12.62 +/- 0.50 min vs. $16.61+/-$ $1.28 \mathrm{~min}$ in the 5 -Fr and 6-Fr groups, respectively; $\mathrm{p}$ $=0.005)$ and, procedure time in the $5-\mathrm{Fr}$ group (38.74 $+/-1.27 \mathrm{~min}$ vs. $46.03+/-2.86 \mathrm{~min}$ in the 5 -Fr and 6-Fr groups, respectively; $p=0.023$ ).

\section{DISCUSSION}

Procedural technical variables like artery-to-sheath ratio, heparin use, and duration of compression have been reported to affect the incidence of RAO. Multiple prospective trials showed smaller sheath sizes associated with reducing RAO. ${ }^{10}$

Although the transradial access has become the preferred access site for coronary intervention for many interventional cardiologists, there also was no conclusive data or consensus among experts on the optimal sheath size that is required during the procedure. Some interventional cardiologists favor the 5-Fr system to limit the risk of radial artery spasm or access site bleeding. On the contrary, there are other operators who prefer the 6-Fr system, since it permits the use of larger devices, and/or larger catheter size that offers better catheter mobility techniques. ${ }^{11}$

Numerous single-center studies were conducted to compare the use of 6-Fr catheters to the 5-Fr systems $s^{8,9}$ with subsequent meta-analysis ${ }^{12}$ performed and published in 2015. The results of that concluded that both the 5-Fr and 6-Fr systems have excellent safety profiles. The use of 5-Fr guide catheters however was associated with a lower contrast medium administration, and lower 
bleedings without compromising procedural success or procedure length. ${ }^{12}$

Hamon et al had reported favorable results using a 5-Fr guiding catheter in patients with ACS. ${ }^{13}$ This study, however, focused on the results of the procedural success rate and access site complications. This did not directly correlate the 5-Fr with 6-Fr guide catheters in regard to the total amount of contrast as well as complete fluoroscopy time.

Few of the studies that compared 5-Fr vs 6-Fr guiding catheters for $\mathrm{TCl}$ in terms of fluoroscopy time, procedure time and contrast amount have included ACS patients among their populations, ${ }^{8,9}$ Yet, the number of ACS patients in these studies was very low. Our study has larger number of ACS patients and is the first to our knowledge to compare the use of 5-Fr versus 6-Fr guiding catheters for $\mathrm{TCl}$ in terms of fluoroscopy time, procedure time and contrast amount only in the setting of ACS. We conclude that even during ACS, the use of 5-Fr guide catheters was associated with a significant reduction in the amount of contrast medium administered, fluoroscopy time, without increasing the overall procedure time. Given the excellent outcomes with the 5-Fr system throughout the elective and/or emergent coronary intervention, we believe this potentially could be the go-to choice for interventional cardiologists.

There are certain subsets of patients that may gain the highest benefits from using 5-Fr guide catheters. For instance, this could be particularly useful in female patients undergoing coronary intervention, as women tend to have on average a smaller radial artery compared to men, which results in a higher likelihood of trauma to the vessel wall when using a 6-Fr catheter compared to 5-Fr guide catheter. ${ }^{14,15}$ Another subgroup of patients that may derive greater benefits from using smaller guide catheters are patients with chronic kidney disease who present with ACS as our study showed lower contrast amount used with 5-Fr catheters compared to 6-Fr catheters. Since it is well known that a lower contrast medium is associated with a lower risk of contrast-induced nephropathy (CIN), consequently, the use of 5-Fr catheters ideally can result in a lower chance of acute renal failure in patients with chronic kidney disease or when recurring procedure is warranted.
Our findings have demonstrated that the use of 5-Fr catheters system in $\mathrm{TCl}$ has led to a lower contrast amount despite being used during ACS (NSTEMI, STEMI, and UA), in comparison to 6-Fr system. In addition to the many other benefits for using a $5-\mathrm{Fr}$ sheath and guiding catheter, it has a favorable effect on vascular access complications.

Based on our study results we correspond with the previous conclusions indicating that a learning curve is crucial for a successful round of the 5-Fr strategies. In addition to the constant advancements and evolution of the guiding catheters, it is vital for all interventional cardiologists to familiarize themselves with the various shapes and sizes of guiding catheters.

There is no dispute that using 5-Fr guiding catheters for $\mathrm{TCl}$ still have some limitations especially when more intricate interventions are needed like $\mathrm{PCl}$ to the left main coronary artery, or when kissing balloon strategy is warranted for bifurcation lesions (in this case the 5-Fr catheter size may not be feasible, and a larger catheter size will additionally be required). Another disadvantage of using 5- $\mathrm{Fr}$ catheters is the lack of backup support of the catheter, which may not hold enough for certain coronary intervention procedures.

We concur with Alberto Polimeni et al's conclusion that one significant advantage of the 5 -Fr system is that it can be engaged deeply and selectively into coronary arteries, permitting an influx in the rate of successful stent implantation in a complex coronary anatomy. ${ }^{12}$

\section{LIMITATIONS}

This study is designed retrospectively, so it was not possible to ascertain the post procedure complications; however, there were no major complications mentioned in the charts. Furthermore, due to the retrospective design of this study it was not possible to look for and ascertain various factors that led to a sample size discrepancy between the 5-Fr and 6-Fr groups which might affect our results. These factors include operator preference/ experience, patient factors such as BMI, age, gender, prior history of catheter use in same patient, 
anticipation of device use in high risk cases, setting/ availability etc.

\section{CONCLUSION}

$\mathrm{TCl}$ in the settings of ACS is safe and feasible, whether using 5-Fr or 6-Fr catheters. Our study concluded that using 5-Fr catheters for $\mathrm{TCl}$ could be preferred for patients presenting with ACS due to lower amount of contrast medium used and less fluoroscopy and procedure time. However, this is a single center retrospective study so we suggest that large randomized controlled studies are needed.

\section{AUTHOR AFFILIATIONS}

1. Marshall University Joan C. Edwards School of Medicine, Huntington, West Virginia

2. Al-Najah University School of Medicine, Nablus, Palestine

3. Misr University for Science and Technology, Giza Governorate, Egypt

\section{REFERENCES}

1. Campeau L. Percutaneous radial artery approach for coronary angiography. Cathet Cardiovasc Diagn. 1989;16(1):3-7.

2. Kiemeneij F, Laarman GJ. Percutaneous transradial artery approach for coronary stent implantation. Cathet Cardiovasc Diagn. 1993;30(2):173-8.

3. Agostoni $P$ et al. Radial versus femoral approach for percutaneous coronary diagnostic and interventional procedures; systematic overview and meta-analysis of randomized trials. J Am Coll Cardiol. 2004;44(2):349-356.

4. Jolly SS et al. Radial versus femoral access for coronary angiography or intervention and the impact on major bleeding and ischemic events: a systematic review and meta-analysis of randomized trials. Am Heart J. 2009;157(1):13240.

5. Uhlemann $M$ et al. The Leipzig prospective vascular ultrasound registry in radial artery catheterization: impact of sheath size on vascular complications. JACC Cardiovasc Interv.
2012;5(1):36-43.

6. Jolly SS et al. Radial versus femoral access for coronary angiography and intervention in patients with acute coronary syndromes (RIVAL): a randomised, parallel group, multicentre trial. Lancet. 2011; 377(9775):1409-1420.

7. Bertrand OF et al. Transradial approach for coronary angiography and interventions: results of the first international transradial practice survey. JACC Cardiovasc Interv. 2010;3(10):102231.

8. Dahm JB et al. A randomized trial of 5 vs. 6 French transradial percutaneous coronary interventions. Catheter Cardiovasc Interv. 2002;7(2):72-76.

9. Gwon $\mathrm{HC}$ et al. A 5Fr catheter approach reduces patient discomfort during transradial coronary intervention compared with a 6Fr approach: a prospective randomized study. J Interv Cardiol. 2006;19(2):141-147.

10. Shantha GPS, Pancholy SB. Comparison of incidence of radial artery occlusion in patients undergoing trans-radial intervention with or without a sheath: a prospective cohort study. Circulation. 2014;130:A13877.

11. Dahm JB, van Buuren F. Transradial percutaneous coronary interventions: indications, success rates \& clinical outcome. Indian Heart J. 2010;62(3):218-220.

12. Polimeni A et al. Clinical and procedural outcomes of 5-French versus 6-French sheaths in transradial coronary interventions. Medicine. 2015;94(52):e2170.

13. Hamon $M$ et al. Mini-invasive strategy in acute coronary syndromes: direct coronary stenting using 5 Fr guiding catheters and transradial approach. Catheter Cardiovasc Interv. 2002;55(3):340-343.

14. Argulian E et al. Gender differences in short-term cardiovascular outcomes after percutaneous coronary interventions. Am J Cardiol. 2006;98(1):48-53.

15. Lansky AJ et al. Gender differences in outcomes after primary angioplasty versus primary stenting with and without abciximab for acute myocardial infarction: results of the Controlled Abciximab and Device Investigation to Lower Late Angioplasty Complications (CADILLAC) trial. Circulation. 2005; 111(13):1611-1618. 